\title{
Can iron be a risk factor for coronary lesions in Kawasaki disease?
}

Kyung Lim Yoon, MD

Department of Pediatrics, Kyung Hee University Hospital at Gangdong, Seoul, Korea

Kawasaki disease $(\mathrm{KD})$ is a systemic vasculitis leading to coronary artery lesions (CAL), the most common cause of acquired heart disease in children in developed countries.

White blood cell count, polymorphonuclear leukocytes, hemoglobin, C-reactive protein (CRP), erythrocyte sedimentation rate, albumin, aspartate aminotransferase, alanine aminotransferase, total bilirubin, sodium, platelet count, N-terminal pro-brain natriuretic peptide, interleukin-6 (IL-6), and IL-10 are effective laboratory predictors for intravenous immunoglobulin (IVIG) resistance and CAL in KD patients. ${ }^{1,2)}$ The initial administration of IVIG $\leq 4.0$ days after symptom onset, oral mucosa alterations, cervical lymphadenopathy, and swelling of the extremities are known clinical predictors of IVIG resistance.)

Anemia commonly occurs in KD patients despite normochromic and normocytic status. The mechanism of anemia in $\mathrm{KD}$ is prolonged active inflammation; however, its correlation with iron deficiency is uncertain. Iron deficiency can occur as an absolute and/or functional deficiency. ${ }^{3)}$ Anemia of inflammation (AI) is the ultimate consequence of these functional disturbances of iron homeostasis and can be seen in cases of acute and chronic infections, inflammatory disorders, and malignancies. Combined forms of iron deficiency anemia (IDA) and AI are typically observed in patients with inflammatory bowel disease (IBD) or gastrointestinal or urogenital malignancies. Anticoagulant or antiplatelet drugs may contribute to iron loss in chronic kidney disease and other chronic diseases. ${ }^{3)}$

The activation of immune cells by infectious agents and auto-antigens initiates and maintains the development of AI by several mechanisms. The excessive production of inflammatory mediators diverts iron to the mononuclear phagocyte system (MPS), rendering it relatively unavailable for erythroid progenitors. Hepcidin antimicrobial peptide (HAMP) is the hormonal negative-feedback regulator of serum iron that limits iron fluxes to the circulation. Increased HAMP levels have been documented in infections, rheumatoid disorders, and IBD in contrast to suppressed levels in IDA. HAMP concentration positively correlates with the extent of inflammation, in relation to iron sequestration in the MPS. In AI, inflammatory markers such as CRP or IL-6 must be considered with altered serum ferritin production and levels. ${ }^{3)}$ Hyperferritinemia ( $>200 \mathrm{ng} / \mathrm{mL}$ ) with decreased transferrin saturation is indicative of $\mathrm{AI}^{3)}$

Hepcidin controls iron metabolism and the pathogenesis of $\mathrm{AI}^{4}{ }^{4)}$ Increased hepcidin levels are correlated with the development of anemia and disease outcomes in patients with $\mathrm{KD}^{5}{ }^{5}$ Huang et al. ${ }^{6}$ reported that hepcidin induces transient anemia and hypoferremia during the acute inflammatory phase of KD. In this study, the hemoglobin levels were lower in pre-IVIG patients than controls and continued to decrease after IVIG treatment. Both the plasma iron levels and total iron-binding capacity (TIBC) were lower in patients with pre- and post-IVIG treatment. The transferrin saturation (iron/TIBC) was lowest prior to IVIG treatment. Several studies have reported that inflammation-induced hepcidin can induce transient anemia and hypoferremia in the acute phase of KD. KD patients often present with hepatitis and jaundice; since hepcidin is produced mainly in hepatocytes, modified hepcidin expression can occur in $\mathrm{KD}^{6}{ }^{6}$ Thus, hepcidin may be helpful in the differential diagnosis of anemias since it is suppressed in IDA
Corresponding author: Kyung Lim Yoon, MD, PhD Department of Pediatrics, Kyung Hee University Hospital at Gandong, 892 Dongnam-ro, Gandonggu, Seoul 05278, Korea

Tel: +82-2-440-7000

Fax: +82-2-440-7175

E-mail: ykr3215@hanmail.net

https://orcid.org/0000-0003-4734-900X

Received: 25 March, 2019

Revised: 3 June, 2019

Accepted: 7 June, 2019
Copyright @ 2019 by The Korean Pediatric Society

This is an open-access article distributed under the terms of the Creative Commons Attribution NonCommercial License (http://creativecommons.org/ licenses/by-nc/4.0/ which permits unrestricted noncommercial use, distribution, and reproduction in any medium, provided the original work is properly cited. 
and elevated in AI.

There is increasing evidence that iron status is associated with coronary artery disease and vasculitis. In the study in this issue ${ }^{7)}$, the authors reported that iron deficiency can predict CAL development in KD. Anemia, iron deficiency, and IDA were significantly associated with CAL compared to other clinical and laboratory predictive findings of CAL. The authors reported that a low ferritin level was highly associated with CAL.

Assuming that the KD patient had already developed IDA, this finding implies that breastfed infants are at higher risk of developing $\mathrm{KD}$ than bottle-fed infants, but evidence of this is lacking. Therefore, iron deficiency in high-risk KD patients may be caused by functional iron deficiency of inflammation rather than absolute iron deficiency. In another study, serum ferritin level was significantly elevated $(\geq 165 \mathrm{ng} / \mathrm{mL}$ ) in nonresponders, a finding that is in contrast to that reported in the current study. The authors of that study found that many nonresponders to IVIG with low-risk scores had high serum ferritin levels.

Before iron or hepcidin can be considered treatment options, further studies including serum hepcidin level as well as hemoglobin and iron indices may be helpful to identify risk factors for CAL among KD patients.

\section{Conflicts of interest}

No potential conflict of interest relevant to this article was reported.

\section{References}

1. Li X, Chen Y, Tang Y, Ding Y, Xu Q, Sun L, et al. Predictors of intravenous immunoglobulin-resistant Kawasaki disease in children: a metaanalysis of 4442 cases. Eur J Pediatr 2018;177:1279-92.

2. Xie T, Wang Y, Fu S, Wang W, Xie C, Zhang Y, et al. Predictors for intravenous immunoglobulin resistance and coronary artery lesions in Kawasaki disease. Pediatr Rheumatol Online J 2017;15:17.

3. Nairz M, Theurl I, Wolf D, Weiss G. Iron deficiency or anemia of inflammation? : Differential diagnosis and mechanisms of anemia of inflammation. Wien Med Wochenschr 2016;166:411-23.

4. Fleming RE, Bacon BR. Orchestration of iron homeostasis. N Engl J Med 2005;352:1741-4.

5. Kuo HC, Yang YL, Chuang JH, Tiao MM, Yu HR, Huang LT, et al. Inflammation-induced hepcidin is associated with the development of anemia and coronary artery lesions in Kawasaki disease. J Clin Immunol 2012;32:746-52.

6. Huang YH, Kuo HC, Huang FC, Yu HR, Hsieh KS, Yang YL, et al. Hepcidin-induced iron deficiency is related to transient anemia and hypoferremia. Int J Mol Sci 2016;17(5). pii: E715. https://doi.org/ 10.3390/ijms17050715.

7. Kim SH, Eun LY. Iron deficiency anemia as a predictor of coronary artery abnormalities in Kawasaki disease. Korean J Ped 2019:62:3016.

8. Yamamoto N, Sato K, Hoshina T, Kojiro M, Kusuhara K. Utility of ferritin as a predictor of the patients with Kawasaki disease refractory to intravenous immunoglobulin therapy. Mod Rheumatol 2015;25: 898-902. 